\title{
A multi-time scale solution strategy for an elastomeric aircraft
}

\author{
Lei Yang ${ }^{\mathrm{a}, \mathrm{b}, *}$, Quan-hua Sun ${ }^{\mathrm{a}, \mathrm{b}}$ \\ a State Key Laboratory of High-Temperature Gas Dynamics, Institute of Mechanics, Chinese Academy of Sciences, Beijing 100190, PR China \\ $\mathrm{b}$ Laboratory for Aerospace Science and Technology, Institute of Mechanics, Chinese Academy of Sciences, Beijing 100190, PR China
}

\section{A R T I C L E I N F O}

\section{Article history:}

Received 18 December 2019

Received in revised form 7 March 2020

Accepted 1 April 2020

Available online 1 June 2020

Communicated by Weiwei Zhang

\section{Keywords:}

Flight dynamic simulation

Multi-time scale

Elastomeric aircraft

Reduced order models

Store separation

\begin{abstract}
A B S T R A C T
This paper develops a multi-time scale kinetics solution strategy for the flight dynamic simulation of elastomeric aircraft. The combination of computational fluid dynamics (CFD) solver and reduced-order model is utilized to calculate the aerodynamics of elastic aircraft. CFD is applied to the aerodynamic solution of rigid motion, and reduced-order model is to the elastic deformation. The governing equations of rigid-body dynamics and structure dynamics are decoupled in time-scale, and their time step sizes can be set differently. This method overcomes the problem of large difference in characteristic times between rigid-body dynamics and structure dynamics, and improves the calculation efficiency of CFD based multi-disciplinary coupling solution. For current work, the multi-time scale kinetics solution method is applied to the simulation of store separation problem considering elastic deformation of carrier aircraft. The autoregressive with exogenous input model is employed to establish the generalized aerodynamic model of carrier. By comparison, the time responses of store obtained by the multi-time scale kinetics solution method are in good agreement with those by traditional CFD based multi-disciplinary coupling simulation, which validates high precision of the new method. Furthermore, the calculation amount of the new coupled calculation method is reduced by about an order of magnitude compared with that of the traditional method. The multi-time scale kinetics solution method can provide an effective solution strategy for similar engineering problems.
\end{abstract}

(c) 2020 Elsevier Masson SAS. All rights reserved.

\section{Introduction}

The air-launch-to-orbit is a low-energy and high-flexibility space transportation technology for future, and has attracted broad attention [1]. With this launching approach, large transport airplane (carrier aircraft) provides initial kinetic and potential energies for spacecraft (store), and less fuel is consumed for the spacecraft to enter orbit [2]. However, there are many technical problems in this process, among which the separation of spacecraft is the key issue. Primarily, spacecraft is usually mounted below the wing, and the flow field of the combined aircraft is extremely complicated. Secondly, the separation is a dynamic process, in which strong unsteady aerodynamic interferences can occur between the carrier aircraft and external spacecraft [3]. Moreover, for the large transport airplane with a high aspect ratio, the deformation of the wing should be considered, and the aeroelastic responses of the carrier can have an impact on spacecraft. As a result, the flight stability of spacecraft is most likely to be influenced by carrier aircraft and other surroundings [4,5].

\footnotetext{
* Corresponding author at: State Key Laboratory of High-Temperature Gas Dynamics, Institute of Mechanics, Chinese Academy of Sciences, Beijing 100190, PR China. E-mail address: yanglei@imech.ac.cn (L. Yang).
}

For the store separation technology, seeking safe and reliable separation method is the primary problem to be considered [6-8], and a great deal of numerical calculations and experiments are needed. In recent years, with the advances of computer technology, the dynamic grid technology and CFD have been rapidly developed, and the CFD/RBD coupled method has been widely applied to flight simulation and store separation of rigid aircraft [9-13]. However, less work has been done for the elastomeric vehicles. In our previous research, structure dynamics is coupled in the simulation system, and the CFD/CSD/RBD coupling method is developed and utilized in the simulation of the store separation process $[4,14,15]$.

The CFD/CSD/RBD coupling method has high calculation accuracy. Nevertheless, the huge amount of calculation is the primary factor that prevents it from being extended to engineering applications. During the CFD/CSD/RBD coupling simulation, the majority of CPU time is taken up by solving the Naiver-Stokes (N-S) equation and processing dynamic grid. Therefore, there are two ways to improve the simulation efficiency: one is to replace CFD method by an efficient aerodynamic model [16], such as the ROM [17,18]; the other is to reduce the call number of CFD.

At present, the ROM is excellently suitable for the linear problem [19-21] and most of the nonlinear problem [22-24]. However, for store separation problem, the unsteady interfering aerodynamic forces between store and carrier aircraft are complex. Solving the 


\section{List of abbreviations}

ALE Arbitrary Lagrangian-Eulerian

ARX Autoregressive Model with Exogenous Input

CFD Computational Fluid Dynamics

CSD Computational Structure Dynamics

GFSI Generalized Fluid-Structure Interaction
LU-SGS Lower-Upper Symmetric Gauss-Seidel method

RBD Rigid-Body Dynamics

ROM Reduced-Order Model

SILMS Semi-Implicit Linear Multi-Step Scheme

MTS Multi-Time Scale multibody dynamics problems only with the aerodynamic modeling method is difficult for us. Thus, CFD method cannot be abandoned.

Increasing physical time step size is an effective way to reduce the iteration number of CFD. For the store separation problem, the physical time is less than $2 \mathrm{~s}$. The calculation amount of the CFD/RBD method is acceptable for engineering in terms of current computer technology. Compared with the CFD/RBD coupled simulation, the computational efficiency of the CFD/CSD/RBD coupled simulation is limited by the time step size. The natural frequency of the flight mechanic mode of aircraft is 0.005 to $1 \mathrm{~Hz}$, and that of the elastic mode ranges from 1 to $1000 \mathrm{~Hz}$. During the CFD/RBD simulation, the choice of time step size is based on the flight mechanic mode. Only 20 to 40 unsteady time iterations are required in one rigid body dynamic cycle. However, the time step size of the CFD/CSD/RBD simulation is based on the elastic mode and is greatly reduced. It means that thousands of unsteady time iterations are carried out in one period of flight mechanics and the calculation amount is huge. For the study of CFD/CSD/RBD coupled simulation, if time step size is based on the flight mechanic mode, it will result in poor accuracy of elastic deformation or divergence of the structural dynamic equation. Then, is there a way to magnify time step size for the flight dynamic simulation of the elastic aircraft with high efficiency and precision?

An answer is the combination of ROM and CFD. For the aircraft structure dynamic simulation, on one hand, as the elastic deformations of conventional aircraft are smaller than its reference length within its flight envelope, the variation of generalized aerodynamic forces is linear due to the elastic deformation. On the other hand, smaller characteristic times lead to smaller time step size, the aerodynamic solver is called frequently. Then the generalized aerodynamic forces can be established by the linear ROM with high efficiency, precision and robustness. For the flight dynamics, in order to accurate unsteady interference aerodynamics better, unsteady CFD method is employed. In the simulation, as the aerodynamic solvers of rigid-body dynamics and structure dynamics are different, the time step sizes can be set differently. When time step size of rigid-body dynamics simulation is much larger than that of the structure dynamics, the simulation efficiency will increase. According to this idea, the multi-time scale CSD/RBD coupled simulation method is developed in this research. This method takes advantages of both CFD and ROM methods, and overcomes the problem of large difference in characteristic times between rigid-body dynamics and structure dynamics, improving the calculation efficiency of CSD/RBD coupled simulation.

In this paper, the solution strategy of the multi-time scale simulation method is introduced in detail. The ARX model is employed to establish the unsteady aerodynamic ROM of elastic aircraft. Focusing on the store separation problem considering elastic deformation of carrier, the time responses are calculated with the $\mathrm{CFD} / \mathrm{CSD} / \mathrm{RBD}$ method and the multi-time scale $\mathrm{ROM} / \mathrm{CFD} / \mathrm{CSD} / \mathrm{RBD}$ method (or MTS ROM/CFD/CSD/RBD, for short) respectively. By comparing and analyzing the calculation results of the two methods, the computational accuracy and efficiency of the MTS ROM/CFD/CSD/RBD method can be verified.

\section{Coupling calculation method}

\subsection{Dynamic solution strategy}

For the present research, the combination of ROM and CFD is adopted in the CSD/RBD coupled simulation. The weak coupling solution method is utilized. In this approach, the rigid body dynamic responses are calculated by the CFD/RBD method and the time step size is determined by flight mechanic modes. Meanwhile, the elastic deformation of aircraft is simulated by the ROM/CSD method, and time step size is based on the highest order of elastic modes. As the time step size of CFD/RBD simulation is much larger than that of ROM/CSD, there are a number of ROM/CSD iterations in one CFD/RBD time step, and the flow fields, displacement and attitude angle of the aircraft will be frozen during the ROM/CSD simulation.

For convenience, the solving process is shown in Fig. 1, in which $k$ means current time step, and the time step sizes of CFD/RBD and ROM/CSD are expressed as $n \cdot t$ s and ts respectively. In the simulation system, the system physical time step size is based on $t s$, and the flow field is solved once when the physical time step is iterated for $n$ times. The program flow can be explained as below.

Firstly, the simulation begins, and the program input file, computational grid, structural modal and aerodynamic models are read respectively.

Secondly, CFD/CSD/RBD coupled simulation runs for $m$ steps to accurately calculate the effect of the initial excitation on the dynamic response of the structure. The time step size is $t s^{\prime}\left(t s^{\prime}\right.$ can be different from $t s$ ).

Thirdly, in the No. $m+1$ step, determine whether the number of computation steps $m+1$ is an integer multiple of $n$. If so, the CFD/CSD/RBD coupling method will be used to calculate the flow field, elastic deformation and rigid body motion. The time step size of CFD/RBD is $n \cdot t s$, and that of the CFD/CSD is ts. If not, the current flow field will be frozen, and ROM/CSD method will be employed to solve the elastic deformation of aircraft, the time step size is $t s$.

Fourthly, determine whether the next step $k+1$ is an integer multiple of $n$. If so, the generalized displacement will be converted into real displacement of grid nodes by modal matrix, and the real displacements will be superimposed on CFD grid. If not, keep as it is.

Finally, at the end of each physical time step, dynamic response parameters are outputted. The program will be prepared for the next iteration.

The coupling simulation program is improved from the Generalized Fluid-Structure Interaction (GFSI) code developed by our group [14,15]. There are several simulation modules in the program. Below flowchart introduces the governing equations and computing methods of the modules respectively.

\subsection{CFD module}

The CFD module is applied to calculate the aerodynamic forces of the aircraft. The governing equations are unsteady, threedimensional Navier-Stokes equations, and are described by the Arbitrary Lagrangian-Eulerian (ALE) method. The conservation form 


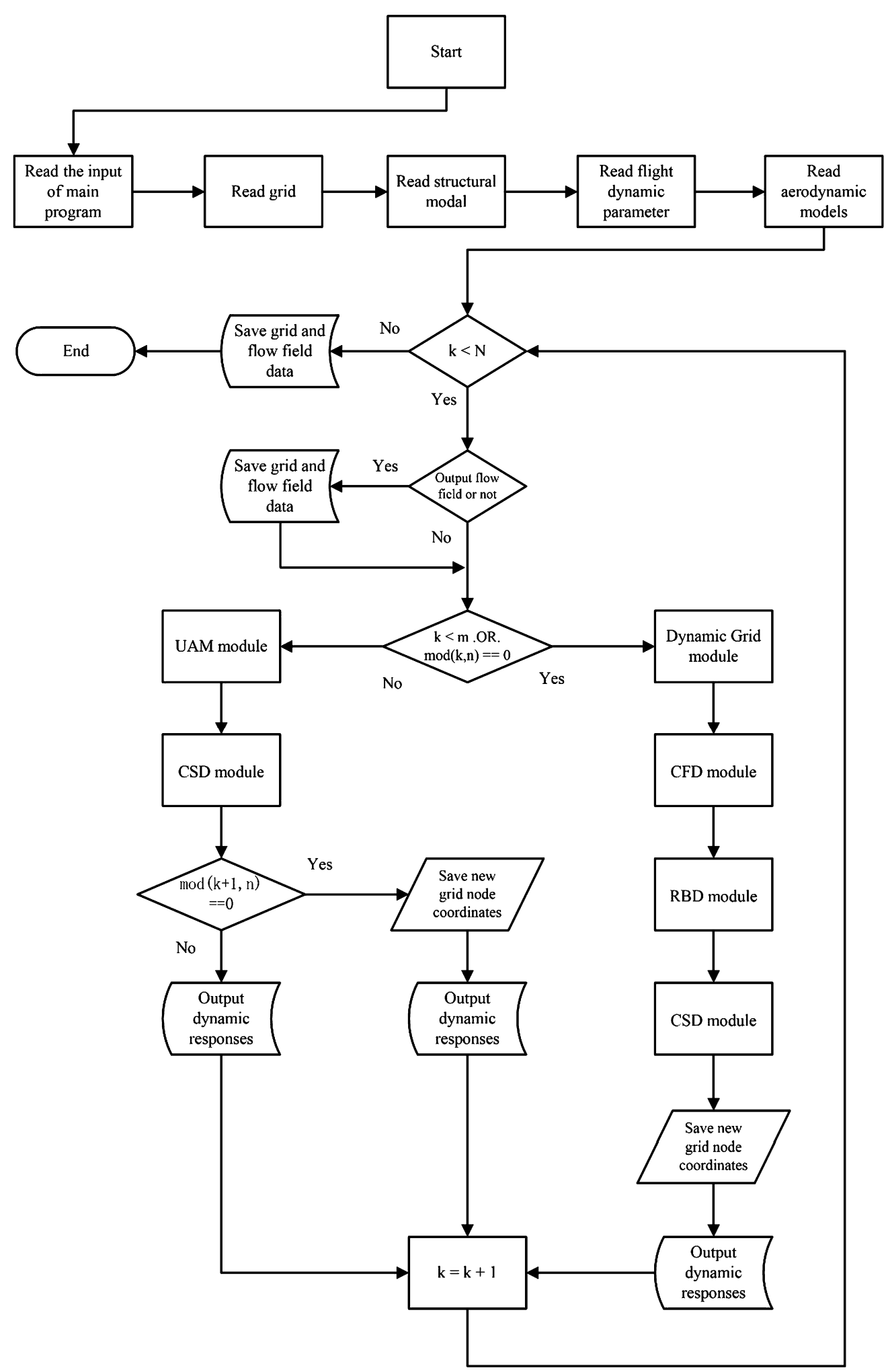

Fig. 1. Flow diagram of MTS ROM/CFD/CSD/RBD simulation method.

of $\mathrm{N}-\mathrm{S}$ equations is written in the earth-fixed rectangular coordinate system as follows [25-27]:

$\frac{\partial}{\partial t} \iiint_{\Omega} \mathbf{Q d} V+\iint_{\partial \Omega} \mathbf{F}\left(\mathbf{Q}, \mathbf{V}_{\text {grid }}\right) \cdot \mathbf{n d} S=\iint_{\partial \Omega} \mathbf{F}^{V}(\mathbf{Q}) \cdot \mathbf{n d} S$

where $\mathbf{Q}$ denotes the vector of conservative variable; $\mathbf{V}_{\text {grid }}$ is velocity vector of a control volume; and $\mathbf{n}$ is the normal vector of boundary face of a control volume. $\mathbf{F}\left(\mathbf{Q}, \mathbf{V}_{\text {grid }}\right)$ and $\mathbf{F}^{V}(\mathbf{Q})$ represent inviscid flux vector and viscous flux vector respectively. $\Omega$ and $\partial \Omega$ refer to control volume and the boundary of control volume respectively.

The state equations of ideal gas are expressed as follows [25].

$$
P=(\gamma-1)\left[e_{0}-\frac{1}{2} \rho(\|\mathbf{V}\|)^{2}\right]
$$


$T=\frac{P}{\rho R}$

where $\mathbf{V}, P, \rho$ and $T$ are velocity vector, pressure, density and temperature respectively. $\gamma$ denotes the specific heat ratio of the gas. $e_{0}$ is the total energy per unit volume of incoming flow, and can be expressed as the sum of the internal energy $e$ and the kinetic energy per unit volume: $e_{0}=e+\frac{1}{2} \rho(\|\mathbf{V}\|)^{2}$.

In the CFD module, the Roe, Steger-Warming, Van Leer and AUSM series numerical methods are available. And the laminar flow model, S-A turbulence model and SST turbulence model can be selected to solve the $\mathrm{N}-\mathrm{S}$ equations. In this paper, $\mathrm{S}-\mathrm{A}$ turbulence model is employed to enclose the $\mathrm{N}-\mathrm{S}$ equations. The dual-time stepping method is used to solve the governing equations. The Lower-Upper Symmetric Gauss-Seidel method (LU-SGS) and Symmetric Gauss-Seidel method can be chosen for the pseudo time marching.

\subsection{CSD module}

When the deformation of elastic body is much smaller than the reference length, the elastic deformation can be regarded as linear and expressed by mode superposition method. Derived from Lagrange equations and the principle of virtual work, the structure dynamic equations based on mode superposition method can be expressed as:

$\mathbf{M} \ddot{\xi}+\mathbf{G} \dot{\xi}+\mathbf{K} \xi=\mathbf{Q}$

where, M means generalized mass matrix; G denotes generalized structural damping matrix; $\mathbf{K}$ is generalized stiffness matrix; and $\mathbf{Q}$ represents generalized force.

When the first $N$ order modes are considered, the elastic displacement vector can be written as:

$\mathbf{w}(x, y, z, t)=\sum_{i=1}^{N} \Phi_{i}(x, y, z) \xi_{i}(t)$

$\mathbf{w}(x, y, z, t)$ is the elastic displacement vector of the structural deformation; $\Phi_{i}(x, y, z)$ is the $i$ th-order mode; and $\xi_{i}(t)$ is generalized coordinate of the $i$ th-order mode. The state space form of the structure dynamic equations is as follows:

$\dot{\mathbf{X}}=\mathbf{A} \cdot \mathbf{X}+\mathbf{B} \cdot \mathbf{Q}(\mathbf{x}, t)$

$\mathbf{A}=\left[\begin{array}{cc}\mathbf{I} & \mathbf{0} \\ -\mathbf{M}^{-1} \mathbf{K} & -\mathbf{M}^{-1} \mathbf{G}\end{array}\right]$,

$\mathbf{B}=\left[\begin{array}{c}\mathbf{0} \\ \mathbf{M}^{-1}\end{array}\right], \mathbf{x}=\left[\xi_{1}, \xi_{2}, \cdots, \xi_{N}, \dot{\xi}_{1}, \dot{\xi}_{2}, \cdots, \dot{\xi}_{N}\right]^{T}$

The structure dynamic equations are calculated by the second order Semi-Implicit Linear Multi-step Scheme (SILMS) [28].

\subsection{RBD module}

Derived from the momentum theorem and the moment of momentum theorem, the six degrees of freedom equations of aircraft in the body-fixed coordinate can be expressed as:

$$
\left\{\begin{array}{c}
F_{x}=m(\dot{u}-v r+w q) \\
F_{y}=m(\dot{v}-w p+u r) \\
F_{z}=m(\dot{w}-u q+v p) \\
M_{x}=\dot{p} I_{x}-I_{x z}(\dot{r}+p q)-\left(I_{y}-I_{z}\right) q r \\
M_{y}=\dot{q} I_{y}-I_{x z}\left(r^{2}-p^{2}\right)-\left(I_{z}-I_{x}\right) r p \\
M_{z}=\dot{r} I_{z}-I_{x z}(\dot{p}-q r)-\left(I_{x}-I_{y}\right) p q
\end{array}\right.
$$

where, $\mathbf{F}$ is total external force vector; $m$ is the mass of aircraft; $\mathbf{v}=\left[\begin{array}{lll}u & v & w\end{array}\right]^{T}$ is centroid velocity vector; $\boldsymbol{\omega}=\left[\begin{array}{ccc}p & q & r\end{array}\right]^{T}$ denotes angular velocity vector; $\mathbf{M}$ represents total moment vector; and $\mathbf{h}$ means the moment of the momentum vector of aircraft. When the first derivative terms are moved to the left side of Eq. (7), the governing equations can be expressed as:

$$
\left\{\begin{array}{c}
m \dot{u}=F_{x}+m v r-m w q \\
m \dot{v}=F_{y}+m w p-m u r \\
m \dot{w}=F_{z}+m u q-m v p \\
\left(I_{x} I_{z}-I_{x z}^{2}\right) \dot{p}=I_{z} M_{x}+I_{x z} M_{z}+I_{z} I_{x z} p q-I_{x z}^{2} q r \\
+\left(I_{y}-I_{z}\right) I_{z} q r+\left(I_{x}-I_{y}\right) I_{x z} p q \\
I_{y} \dot{q}=M_{y}+I_{x z}\left(r^{2}-p^{2}\right)-\left(I_{z}-I_{x}\right) r p \\
\left(I_{x} I_{z}-I_{x z}^{2}\right) \dot{r}=I_{x z} M_{x}+I_{x} M_{z}+I_{x z}^{2} p q-I_{x} I_{x z} q r \\
+\left(I_{x}-I_{y}\right) I_{x} p q+\left(I_{y}-I_{x}\right) I_{x z} q r
\end{array}\right.
$$

The rigid body motion equations are given as follows:

$$
\left\{\begin{array}{c}
\dot{u}_{G}=\cos \theta \cos \psi \cdot u+(-\sin \psi \cos \varphi+\sin \varphi \sin \theta \cos \psi) v \\
+(\sin \psi \sin \varphi+\cos \varphi \sin \theta \cos \psi) w \\
\dot{v}_{G}=\cos \theta \sin \psi \cdot u+(\cos \psi \cos \varphi+\sin \varphi \sin \theta \sin \psi) v \\
+(-\cos \psi \sin \varphi+\cos \varphi \sin \theta \sin \psi) w \\
\dot{w}_{G}=-\sin \theta \cdot u+\cos \theta \sin \varphi \cdot v+\cos \theta \cos \varphi \cdot w \\
\dot{\varphi}=p+\sin \varphi \tan \theta \cdot q+\cos \varphi \tan \theta \cdot r \\
\dot{\theta}=\cos \varphi \cdot q-\sin \varphi \cdot r \\
\dot{\psi}=(\sin \varphi \cdot q+\cos \varphi \cdot r) / \cos \theta
\end{array}\right.
$$

where, $\left[\begin{array}{lll}\varphi & \theta & \psi\end{array}\right]^{T}$ and $\left[\begin{array}{lll}\dot{\varphi} & \dot{\theta} & \dot{\psi}\end{array}\right]^{T}$ are the attitude angle and attitude rate of aircraft respectively, and $\left[\begin{array}{lll}\dot{u}_{G} & \dot{v}_{G} & \dot{w}_{G}\end{array}\right]^{T}$ denotes the velocity of aircraft in the earth-fixed coordinate system.

In the RBD module, the six degrees of freedom dynamic equations and rigid body motion equations are solved by the second order SILMS.

\subsection{ROM module}

In this paper, ARX model is used to build the generalized aerodynamic force model of the elastic aircraft. For the MIMO system (Multiple-input multiple-output system), ARX model is given as follows [29,30]:

$\mathbf{y}(k)=\sum_{i=1}^{n a} \mathbf{A}_{i} \mathbf{y}(k-i)+\sum_{i=0}^{n b-1} \mathbf{B}_{i} \mathbf{u}(k-i)$

where $\mathbf{y}$ means output parameter vector and $\mathbf{u}$ means input parameter vector of the model. In this study, the output and input parameter vectors are the generalized aerodynamic force and the generalized displacement of elastic carrier aircraft respectively. Thus, $\mathbf{y}=\mathbf{f}_{a}$ and $\mathbf{u}=\boldsymbol{\xi}$.na and $n b$ are the delay order of input and feedback of the model respectively, which are determined by the user to minimize the model error between training samples and model results.

Defined the state vectors $\mathbf{x}_{\mathbf{a}}=\left[\mathbf{f}_{a}(k-1), \ldots, \mathbf{f}_{a}(k-n a), \boldsymbol{\xi}(k-\right.$ $1), \ldots, \xi(k-n b+1)]^{T}$ and $\mathbf{x}_{b}=[\boldsymbol{\xi}(k)]^{T}$. Eq. (10) can be expressed as:

$$
\left\{\begin{array}{c}
\mathbf{x}_{a}(k+1)=\tilde{\mathbf{A}}_{a} \mathbf{x}_{a}(k)+\tilde{\mathbf{B}}_{a} x_{b}(k) \\
\mathbf{f}_{a}(k)=\tilde{\mathbf{C}}_{a} \mathbf{x}_{a}(k)+\tilde{\mathbf{D}}_{a} x_{b}(k)+\mathbf{f}_{a 0}
\end{array}\right.
$$

where $\mathbf{f}_{a 0}$ is static aerodynamic coefficient matrix. $\tilde{\mathbf{A}}_{a}, \tilde{\mathbf{B}}_{a}, \tilde{\mathbf{C}}_{a}$ and $\tilde{\mathbf{D}}_{a}$ are undetermined coefficient matrices of the model: 
$\tilde{\mathbf{A}}_{a}=\left[\begin{array}{cccccccccc}A_{1} & A_{2} & \cdots & A_{n a-1} & A_{n a} & B_{1} & B_{2} & \cdots & B_{n b-2} & B_{n b-1} \\ I & 0 & \cdots & 0 & 0 & 0 & 0 & \cdots & 0 & 0 \\ \vdots & I & \cdots & 0 & 0 & 0 & 0 & \cdots & 0 & 0 \\ \vdots & \vdots & & \vdots & \vdots & \vdots & \vdots & & \vdots & \vdots \\ 0 & 0 & \cdots & I & 0 & 0 & 0 & \cdots & 0 & 0 \\ 0 & 0 & \cdots & 0 & 0 & 0 & 0 & \cdots & 0 & 0 \\ 0 & 0 & \cdots & 0 & 0 & I & 0 & \cdots & 0 & 0 \\ 0 & 0 & \cdots & 0 & 0 & 0 & I & \cdots & 0 & 0 \\ \vdots & \vdots & & \vdots & \vdots & \vdots & \vdots & & \vdots & \vdots \\ 0 & 0 & \cdots & 0 & 0 & 0 & 0 & \cdots & I & 0\end{array}\right]$,

$\tilde{\mathbf{B}}_{a}=\left[\begin{array}{c}\tilde{\mathbf{B}}_{0} \\ 0 \\ 0 \\ \vdots \\ 0 \\ I \\ 0 \\ 0 \\ \vdots \\ 0\end{array}\right]$

$\tilde{\mathbf{C}}_{a}=\left[\begin{array}{llllllllll}A_{1} & A_{2} & \cdots & A_{n a-1} & A_{n a} & B_{1} & B_{2} & \cdots & B_{n b-2} & B_{n b-1}\end{array}\right]$,

$\tilde{\mathbf{D}}_{a}=\left[B_{0}\right]$

Eq. (11) can be written in continuous form:

$\left\{\begin{array}{c}\dot{\mathbf{x}}_{a}(t)=\mathbf{A}_{a} \mathbf{x}_{a}(t)+\mathbf{B}_{a} \mathbf{x}_{b}(t) \\ \mathbf{f}_{a}(t)=\mathbf{C}_{a} \mathbf{x}_{a}(t)+\mathbf{D}_{a} \mathbf{x}_{b}(t)+\mathbf{f}_{a 0}\end{array}\right.$

\section{The dynamic simulation and analysis}

\subsection{The calculation model and condition}

For the current research, the MTS ROM/CFD/CSD/RBD dynamic solution strategy is applied to store separation problem considering elastic deformation of carrier aircraft. In the simulation, the carrier aircraft is simplified as an elastic wing. The AGARD 445.6 wing is employed, which is a standard verification case for dynamic simulation and is widely used [31]. The store aircraft is a rigid delta wing aircraft designed by this paper and shown in Fig. 2. The fuselage of the aircraft is revolution body with a length of $800 \mathrm{~mm}$ and a diameter of $60 \mathrm{~mm}$. The wingspan is $364 \mathrm{~mm}$ and the sweep angle is $70^{\circ}$. The distance between the mass center and the nose is $300 \mathrm{~mm}$. The mass of the aircraft is $4.124 \mathrm{~kg}$; the principal moments of inertia along the $x, y$ and $z$ axes are $0.009 \mathrm{~kg} / \mathrm{m}^{2}, 0.122$ $\mathrm{kg} / \mathrm{m}^{2}$ and $0.126 \mathrm{~kg} / \mathrm{m}^{2}$ respectively; and the inertia products $I_{x y}$, $I_{x z}, I_{y z}$ are $0.0 \mathrm{~kg} / \mathrm{m}^{2}, 0.002 \mathrm{~kg} / \mathrm{m}^{2}, 0.0 \mathrm{~kg} / \mathrm{m}^{2}$ respectively.

Mode superposition method is used to simulate the elastic deformation of carrier, and the first four modes are considered. The frequencies of the first four modes are shown in Table 1 and the formations are shown in Fig. 3.

The digital simulation settings are as follows: the incoming flow Mach number is 0.7 ; the angle of attack is $2^{\circ}$; the flight altitude is $15 \mathrm{~km}$; and the Reynolds number based on the mean aerodynamic chord of the carrier aircraft is 1.58E06.

The unstructured hybrid mesh is used for spatial discretization. The grids nearby the wall are prism cells and the others are tetrahedral cells. The surface grids of the carrier and store are shown in Fig. 4 and Fig. 5 respectively. The mesh dependency study has been carefully carried out in our previous work. The total volume cell number is about 5.4 million.
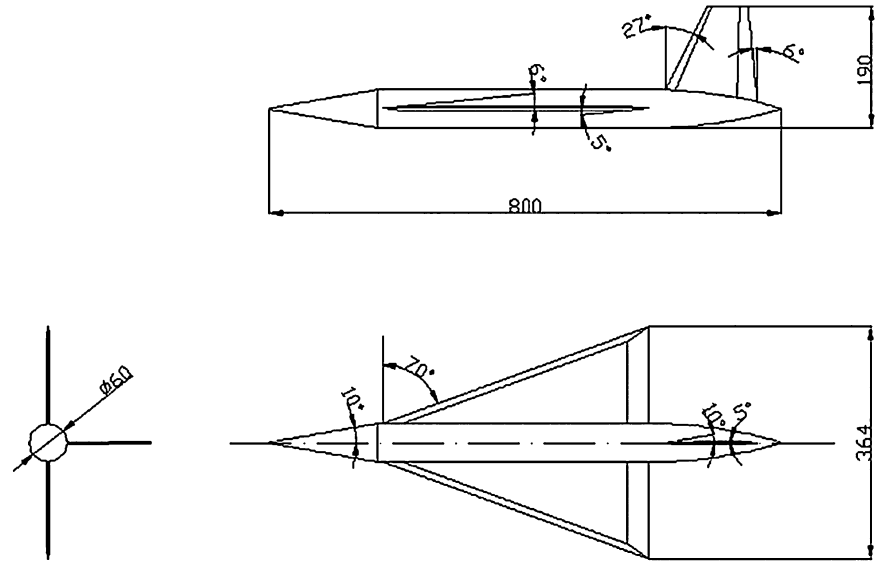

Fig. 2. The three-view drawing of the store.

Table 1

The frequencies of the first four modes.

\begin{tabular}{lll}
\hline Number & Mode & Frequency/Hz \\
\hline Mode 1 & First bending mode & 9.5992 \\
Mode 2 & First torsion mode & 38.1660 \\
Mode 3 & Second bending mode & 48.3482 \\
Mode 4 & Second torsion mode & 91.5448 \\
\hline
\end{tabular}

\subsection{The unsteady aerodynamic model of the elastic carrier}

The unsteady aerodynamic model of the elastic carrier is established in this section. The output and input of the model are generalized aerodynamic force and displacement of the elastic carrier aircraft respectively. Considering the first four modes, the output and input are defined as:

$\mathbf{y}=\left[\begin{array}{llll}f_{a 1} & f_{a 2} & f_{a 3} & f_{a 4}\end{array}\right]^{T}$

$\mathbf{u}=\left[\begin{array}{llll}\xi_{1} & \xi_{2} & \xi_{3} & \xi_{4}\end{array}\right]^{T}$

where $f_{a i}$ means generalized aerodynamic force of the $i$ th order and $\xi_{i}$ is generalized displacement of the $i$ th order.

To obtain a higher accuracy aerodynamic model, the training samples need to contain more kinetic information of the elastic carrier aircraft and be carefully designed. For this research, the training samples are based on "3211" signal and are shown in Fig. 6 . The amplitude ratio of the four modes in training samples is $1: 0.1: 0.01: 0.001$, which is close to that of the actual vibration. Fourier analysis is made for the signal and the results are shown in Fig. 7. The frequency spectrum of the training samples ranges from $0 \mathrm{~Hz}$ to $200 \mathrm{~Hz}$, and the natural frequencies of the first four modes of AGARD445.6 wing are covered. The verifying samples are used to test the accuracy of the model. There should be no intersections between training samples and verifying samples. In this research, the verifying samples are the free vibration responses of the AGARD445.6 wing. The computation conditions are same with the training samples: Mach number is 0.7 , the angle of attack is $2^{\circ}$ and flight altitude is $15 \mathrm{~km}$. The disturbance velocity of the first mode is 0.001 and the others are 0 .

The training samples and verifying samples are calculated by CFD/CSD method. The CFD surface grids of AGARD445.6 wing are the same as Fig. 2, and the volume grid cell number is about 1.8 million. Through modeling and optimization, the delay orders are set as $n a=4$ and $n b=4$. The comparison between training samples and model results are shown in Fig. 8. It can be seen that the model results have good agreement with the training samples. The comparison between verifying samples and model results are shown in Fig. 9, and the model results almost exactly match the verifying samples. Therefore, the model can accurately describe the 


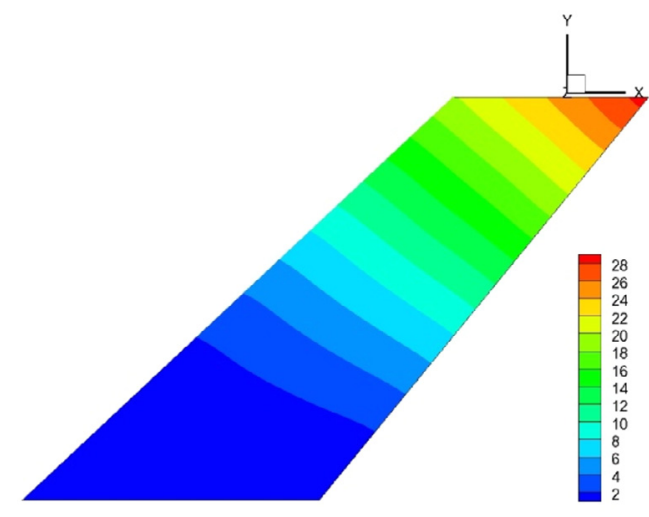

(a) Mode1

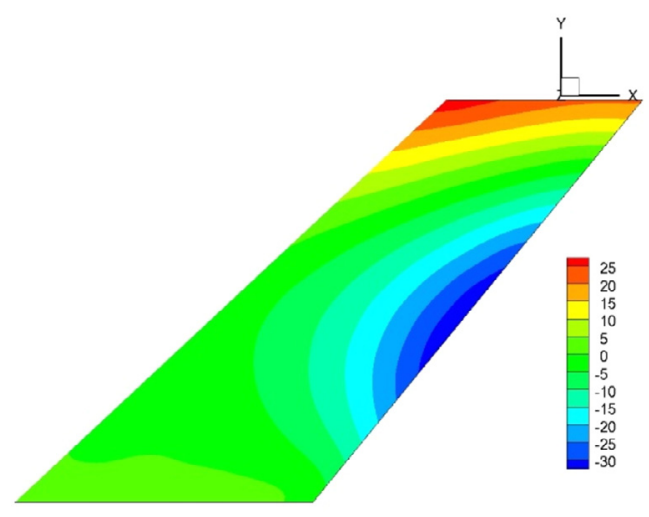

(c) Mode3

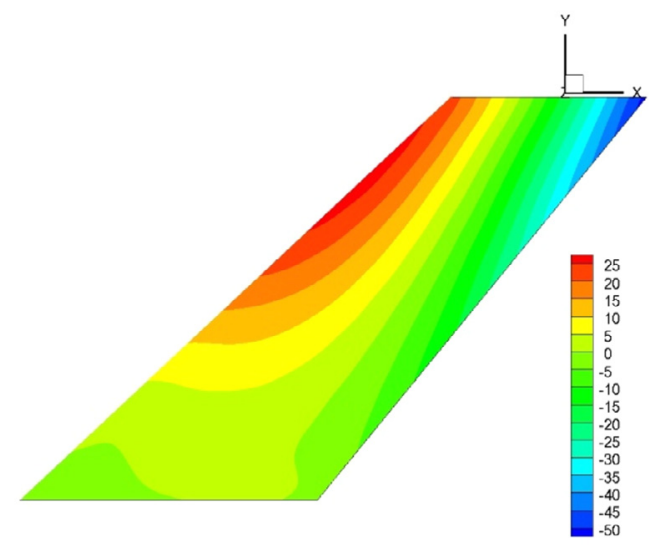

(b) Mode2

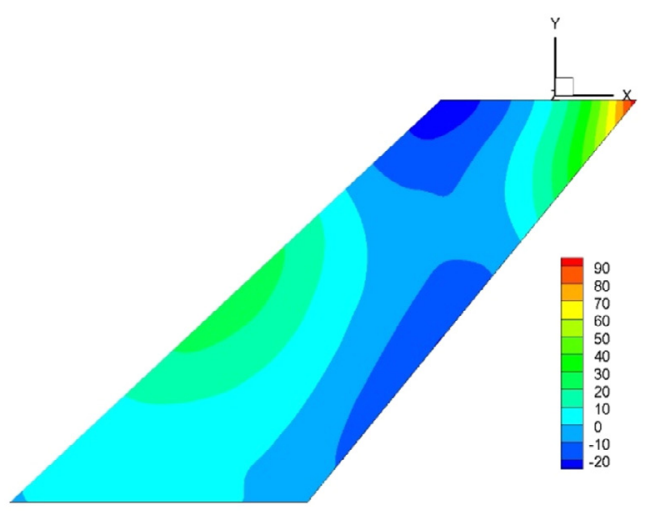

(d) Mode4

Fig. 3. The formations of the first four modes. (For interpretation of the colors in the figure(s), the reader is referred to the web version of this article.)

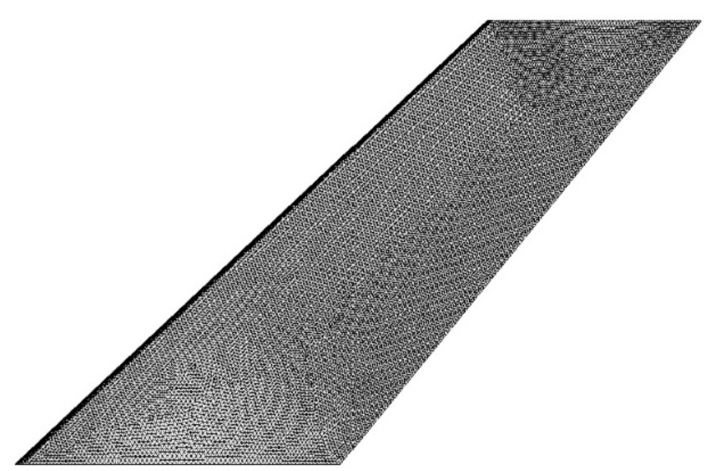

Fig. 4. Surface grids of the AGARD445.6 wing.

aerodynamic force of the elastic carrier aircraft, and can be used to replace the CFD method to calculate the dynamic responses of the elastic deformation.

\subsection{Dynamic simulation of the store separation considering the elastic carrier}

\subsubsection{Time step size convergence study}

For the dynamic simulation, the time step size affects the calculation amount, simulation accuracy and robustness. When it is larger, the simulation accuracy is lower and the robustness is poorer. However, when it is set smaller, the calculation amount

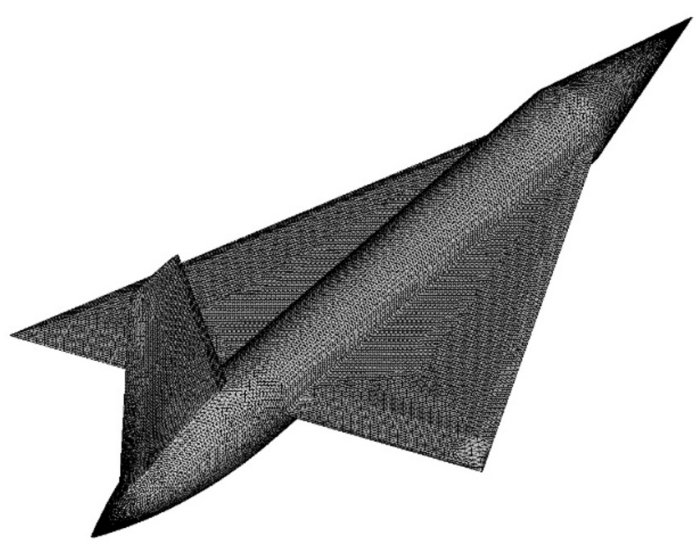

Fig. 5. Surface grids of the store.

will be larger. In order to select an appropriate time step size, its convergence study is illustrated in this section.

For the MTS ROM/CFD/CSD/RBD coupled simulation method developed in this paper, two kinds of time step sizes should be considered. One is the time step size of the CFD/RBD coupled simulation, and the other is that of the ROM/CSD coupled simulation.

For the time step size convergence study of the CFD/RBD coupled simulation, the carrier aircraft is assumed as the rigid body and there is no elastic deformation in the system. The time step 


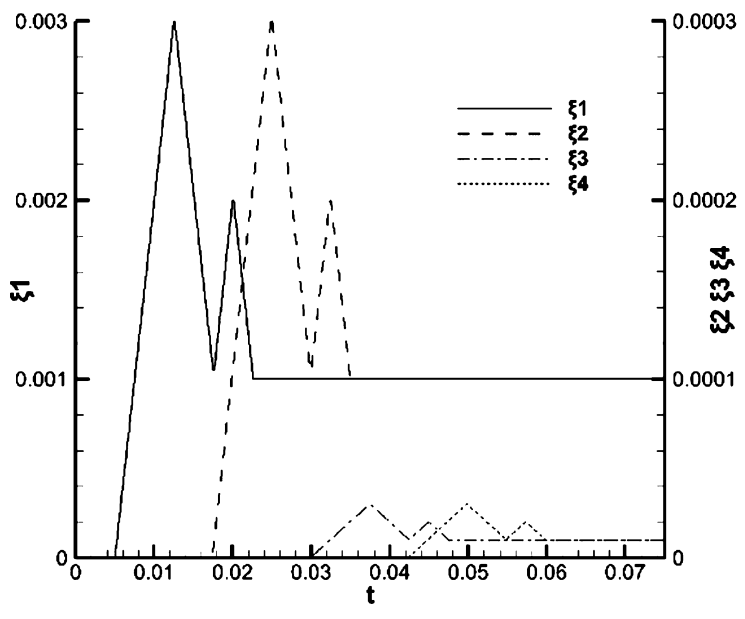

Fig. 6. The training signal.

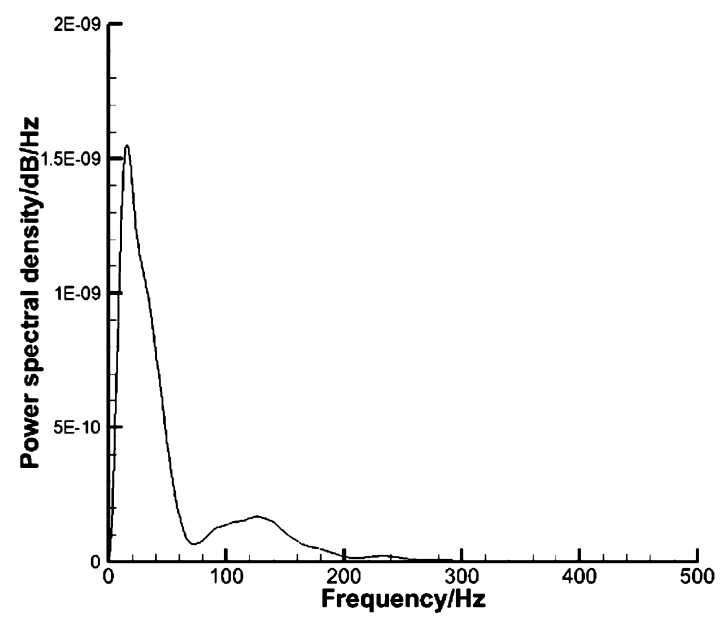

Fig. 7. Fourier analysis of the training signal.

sizes of $0.02 \mathrm{~s}, 0.01 \mathrm{~s}$ and $0.001 \mathrm{~s}$ are considered. For different ones, the rigid body dynamic simulation results of the store are shown in Fig. 10. As we can see, when the time step sizes are set as $0.01 \mathrm{~s}$ and $0.001 \mathrm{~s}$, the time responses are almost the same. When the time step size is increased to $0.02 \mathrm{~s}$, the curves of attitude angle responses gradually depart from the others after the physical time of $0.5 \mathrm{~s}$. Therefore, $0.01 \mathrm{~s}$ is the appropriate time step size for the CFD/RBD coupled simulation. In our previous research, we have the experience that the appropriate time step size of a dynamic system is about one-twentieth or one-fortieth of the period of the highest mode. As shown in Fig. 10, the highest frequency of the dynamic responses is about $5 \mathrm{~Hz}$, and the period is about $0.2 \mathrm{~s}$. It is no doubt that one-twentieth of $0.2 \mathrm{~s}$ is $0.01 \mathrm{~s}$, which agrees well with the above-mentioned conclusion. Consequently, in the current research, the time step size of the CFD/RBD coupled simulation is set as $0.01 \mathrm{~s}$.

In order to determine the time set of ROM/CSD coupled simulation, the time step sizes of $0.0002 \mathrm{~s}, 0.0001 \mathrm{~s}, 0.00005 \mathrm{~s}$ and $0.00001 \mathrm{~s}$ are considered. The carrier aircraft is assumed as an elastic body, and the multi-time scale CFD/CSD/RBD coupled simulation method is employed to calculate the dynamic responses of store separation process. The time step size of the CFD/RBD coupled simulation is $0.01 \mathrm{~s}$. By calculation, we find that the system is instable when the time step size is $0.0002 \mathrm{~s}$. And for the other time sets, the dynamic responses are shown in Fig. 11. As we can see, when the time step is less than $0.0001 \mathrm{~s}$, the time responses of the store and carrier aircraft are almost the same. Therefore, the time step of the ROM/CSD coupled simulation is set as $0.0001 \mathrm{~s}$ in this research.

\subsubsection{Simulation results and analysis}

The dynamic responses of the store considering the interference of the elastic carrier aircraft are simulated by CFD/CSD/RBD coupled method and MTS ROM/CFD/CSD/RBD method respectively. In the simulation, a concentrated load is applied on the elastic carrier before separation. The magnitude of the load equals to the gravity of the store. Then, the static deformation of the wing under the aerodynamic load and concentrated load is calculated with CFD/CSD method. In the time of separation, the concentrated load will disappear instantaneously. The physical time step size is $0.001 \mathrm{~s}$ in the CFD/CSD/RBD simulation. And for the MTS ROM/CFD/CSD/RBD method, the time step sizes of CFD/RBD and $\mathrm{RBD} / \mathrm{CSD}$ coupling calculation are $0.01 \mathrm{~s}$ and $0.0001 \mathrm{~s}$ respectively.

The flow field results at different time calculated by both methods are shown in Fig. 12. As the relative distance between the carrier aircraft and store is farther at $0.94 \mathrm{~s}$, only the surface pressure contour of store is provided in Fig. 12-(e) and Fig. 12-(f). It can be seen that the attitude angles and surface pressure contours calculated by both methods at the same physical time are basically identical.

The rigid body dynamic responses of the store calculated by both methods are shown in Fig. 13. On the whole, the response curves of store obtained by both are almost the same. However, for the attitude angle responses, after the physical time of $0.5 \mathrm{~s}$, the amplitude error increases slightly. The errors of the dynamic responses between the two methods at different physical time are shown in Table 2. As can be seen, the displacement response errors are less than $3 \%$, and the angle response errors are less than $6 \%$. Compared with the small absolute value, the relative error of the yaw angle at $0.5 \mathrm{~s}$ is rather large, which has no value for reference.

The generalized displacement responses of the carrier aircraft are shown in Fig. 14. For their mean and amplitude value, the whole variant trends of both methods are identical. In the first $0.4 \mathrm{~s}$, the phase positions of the two results are almost the same. Nevertheless, as time increases, the phase difference also increases. At the physical time of $0.6 \mathrm{~s}$, the phase difference between the two results is less than $\pi / 2$; but at that of $1.0 \mathrm{~s}$, it increases to $\pi$. Besides, the peak error between the physical time of $0.1 \mathrm{~s}$ to $0.2 \mathrm{~s}$ is a little large. This is mainly because the aerodynamic model of the carrier aircraft ignores the influence of the external store in the MTS ROM/CFD/CSD/RBD method. But the influence of this error on dynamic responses of the store is weak.

To sum up, for the dynamic simulation of store separation considering elastic carrier aircraft interference, the results of MTS $\mathrm{ROM} / \mathrm{CFD} / \mathrm{CSD} / \mathrm{RBD}$ coupled simulation method introduced in this paper are basically consistent with those of the CFD/CSD/RBD method. This method has high precision and can be applied to other similar engineering problems.

\subsubsection{The accumulation error estimates of the simulation}

For unsteady problems, the accumulated error should be considered. For the given allowable value of the total error $S_{\max }$, the allowable number of the time steps can be estimated by the following equation [32,33]:

$n_{\max }=\left(S_{\max } / S_{e r r}\right)^{2}$

In this paper, the CFD code has 2-order numerical scheme and based on the hybrid unstructured grid, the mean ratios of the cell size can be expressed as $\Delta L=\sqrt[3]{V / c_{n}}$. There, $V$ means the volume 


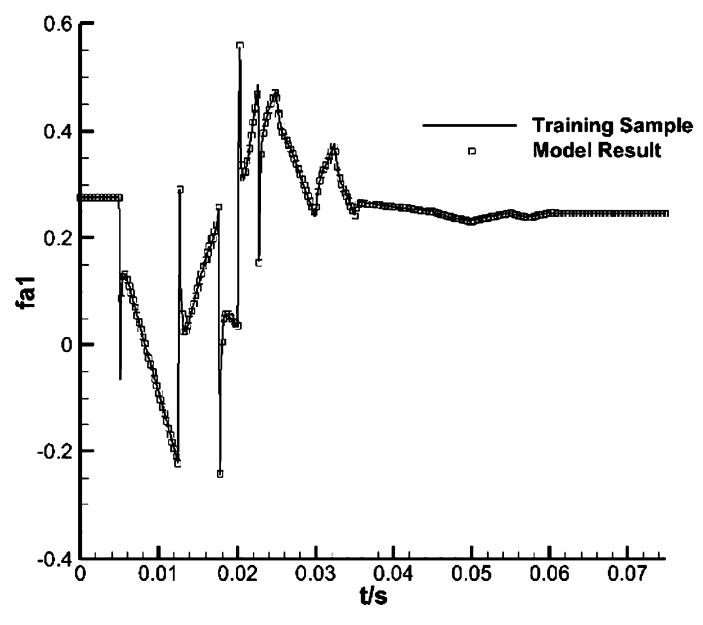

(a) fa1

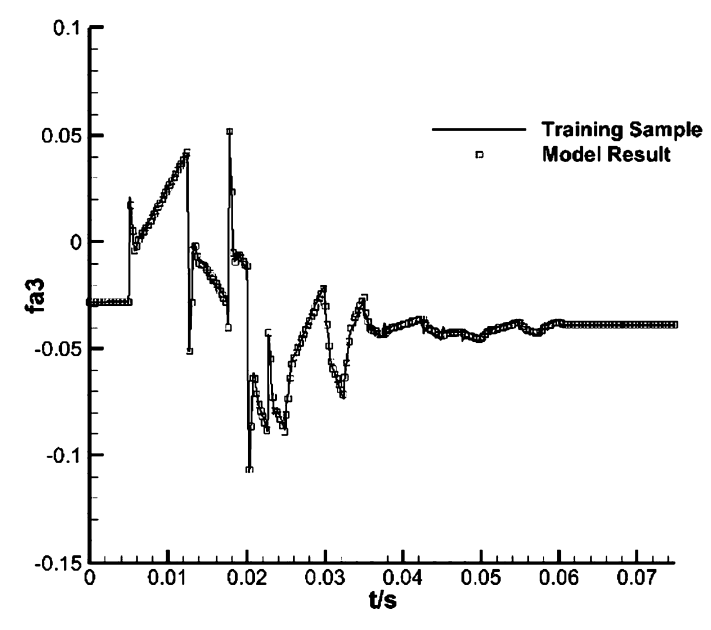

(c) fa3

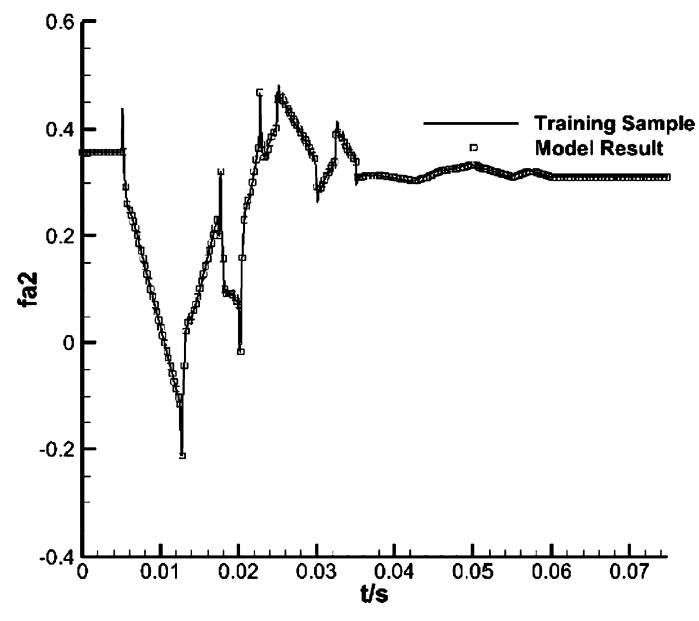

(b) fa2

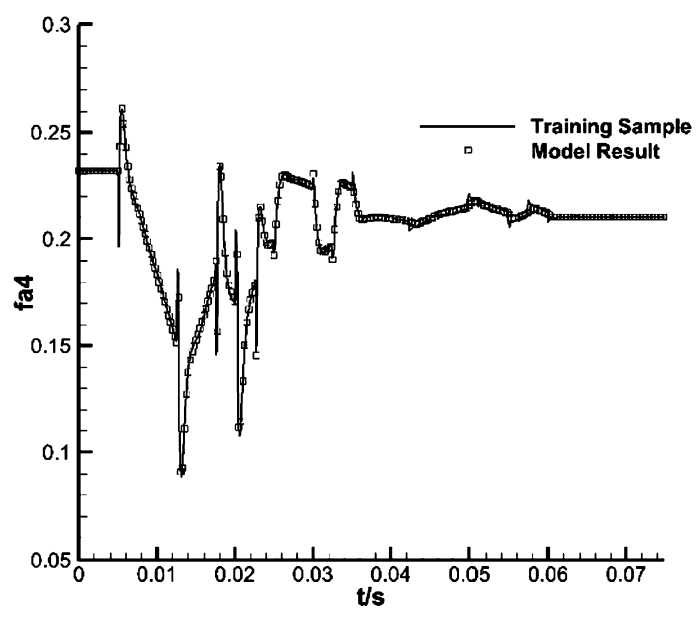

(d) fa4

Fig. 8. The comparison of the generalized aerodynamics forces between training samples and model results.

Table 2

The error of the dynamic responses at different physical time.

\begin{tabular}{llllllll}
\hline $\mathrm{t} / \mathrm{s}$ & Calculation method & $\mathrm{x} / \mathrm{m}$ & $\mathrm{y} / \mathrm{m}$ & $\mathrm{z} / \mathrm{m}$ & $\varphi / \mathrm{rad}$ & $\theta / \mathrm{rad}$ & $\psi / \mathrm{rad}$ \\
\hline \multirow{2}{*}{0.2} & CFD/CSD/RBD results & 0.005863 & 0.000416 & -0.12978 & 0.049089 & -0.03703 & 0.017587 \\
& MTS ROM/CFD/CSD/RBD results & 0.005958 & 0.000423 & -0.13093 & 0.05004 & -0.03639 & 0.017809 \\
& Relative error & $1.62 \%$ & $1.73 \%$ & $0.89 \%$ & $1.94 \%$ & $-1.72 \%$ & $1.27 \%$ \\
\multirow{2}{*}{0.5} & CFD/CSD/RBD results & 0.046501 & -0.0122 & -1.05552 & 0.338345 & -0.0311 & -0.00274 \\
& MTS ROM/CFD/CSD/RBD results & 0.04749 & -0.01261 & -1.05935 & 0.353076 & -0.03098 & -0.00374 \\
& Relative error & $2.13 \%$ & $3.32 \%$ & $0.36 \%$ & $4.53 \%$ & $-0.38 \%$ & $36.47 \%$ \\
\multirow{2}{*}{0.8} & CFD/CSD/RBD results & 0.125901 & -0.03129 & -2.84442 & 0.592505 & -0.05919 & -0.01425 \\
& MTS ROM/CFD/CSD/RBD results & 0.129059 & -0.03125 & -2.85441 & 0.622221 & -0.0568 & -0.01504 \\
& Relative error & $2.51 \%$ & $-0.11 \%$ & $0.35 \%$ & $5.02 \%$ & $-4.03 \%$ & $5.55 \%$ \\
\multirow{2}{*}{1} & CFD/CSD/RBD results & 0.200427 & -0.04515 & -4.50043 & 0.743511 & -0.0675 & -0.0087 \\
& MTS ROM/CFD/CSD/RBD results & 0.204979 & -0.04496 & -4.51429 & 0.788146 & -0.06437 & -0.00853 \\
& Relative error & $2.27 \%$ & $-0.41 \%$ & $0.31 \%$ & $6.00 \%$ & $-4.63 \%$ & $-1.97 \%$ \\
\hline
\end{tabular}

of the domain and $c_{n}$ means the cell number. Then the $S_{\text {err }}$ can be expressed as:

$$
\begin{aligned}
S_{\text {err }}= & \left(\frac{\Delta L}{L_{1}}\right)^{3}+\left(\frac{\Delta L}{L_{2}}\right)^{3}+\left(\frac{\Delta L}{L_{3}}\right)^{3} \\
& =\frac{V}{c_{n}}\left[\left(\frac{1}{L_{1}}\right)^{3}+\left(\frac{1}{L_{2}}\right)^{3}+\left(\frac{1}{L_{3}}\right)^{3}\right]
\end{aligned}
$$

The accumulated error is:

$$
S=\sqrt{n} \times S_{e r r}
$$

The error estimates are shown in Table 3 for different simulation methods. When the allowable value of the total accumulated error is 0.001 , the allowable number of time steps is 1166400 . Because the time steps number is less than 1000, the error is below the given value in this paper. For the MTS ROM/CFD/CSD/RBD sim- 


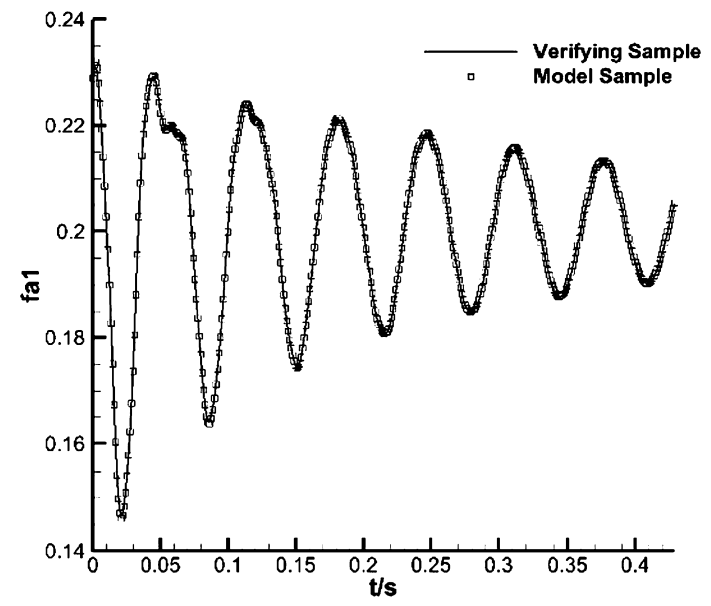

(a) fa1

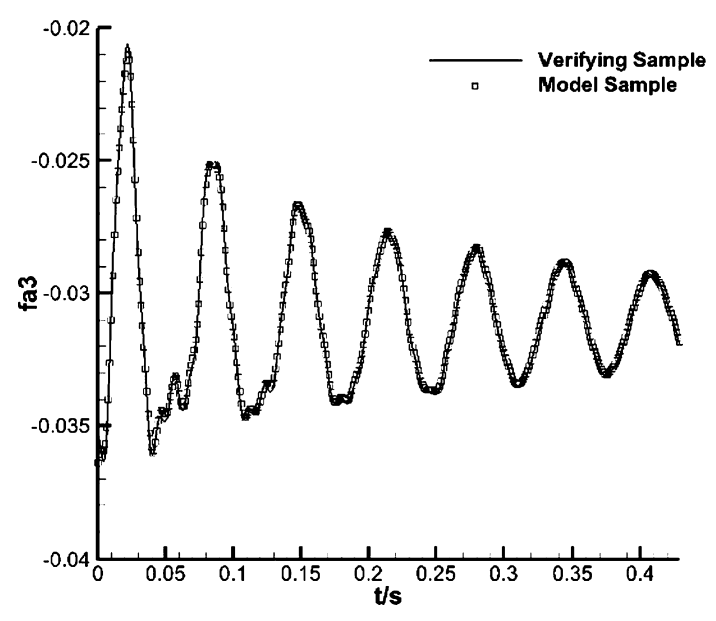

(c) fa3

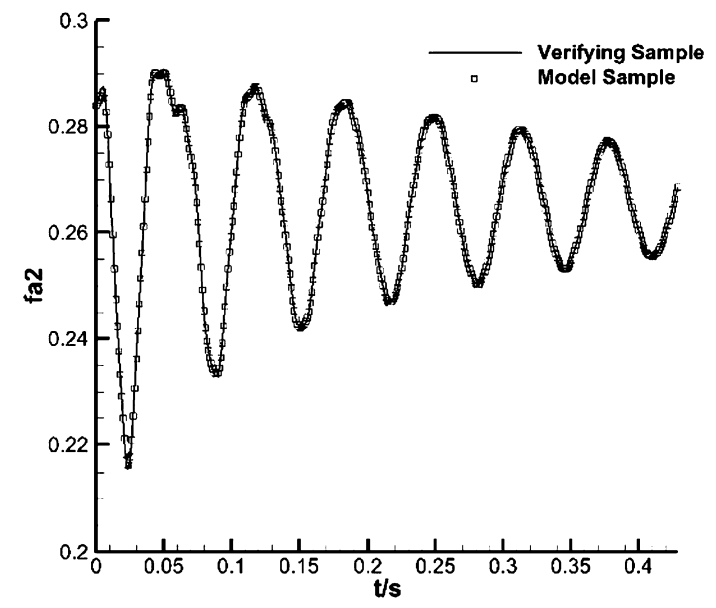

(b) fa2

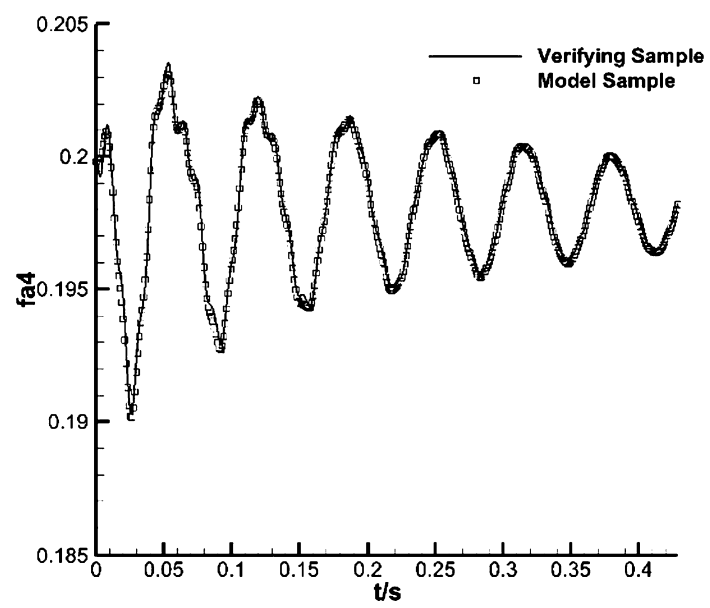

(d) fa4

Fig. 9. The comparison of the generalized aerodynamics forces between verifying samples and model results.

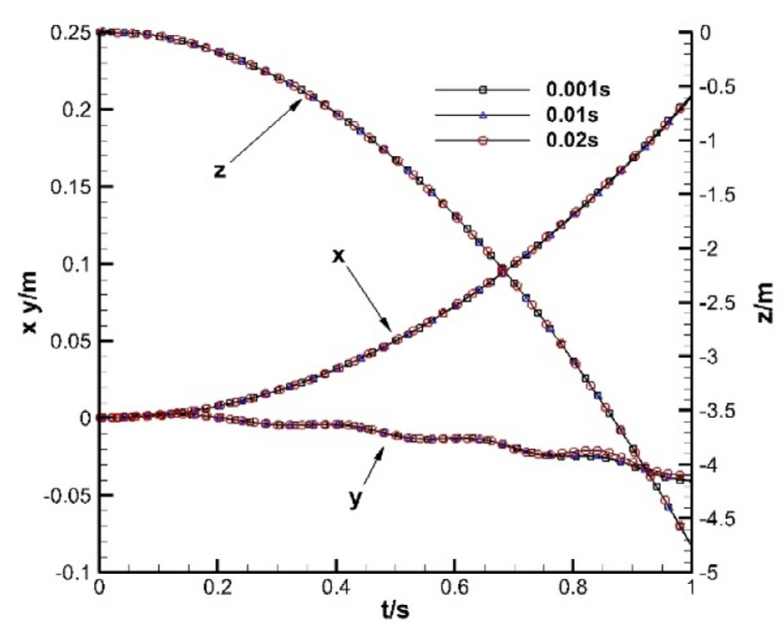

(a) The displacement responses of the store

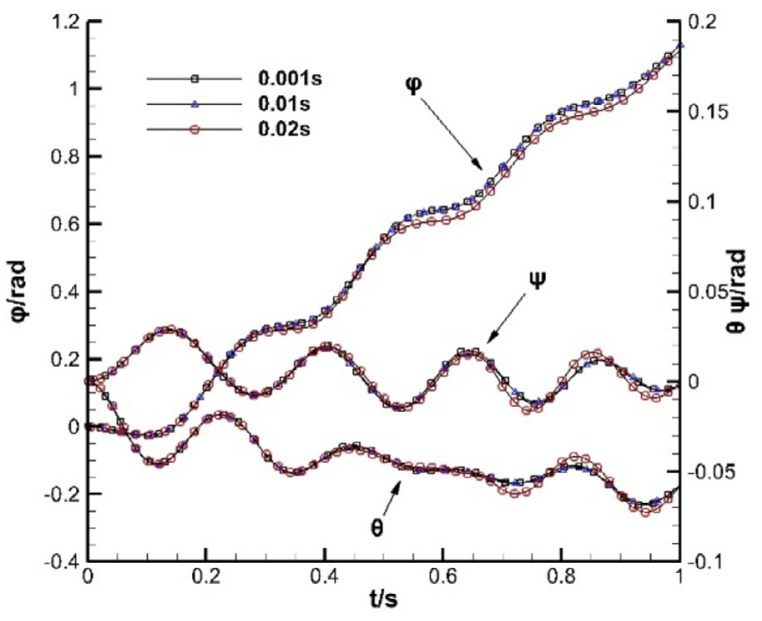

(b) The attitude angle responses of the store

Fig. 10. The dynamic responses of the store for different time step sizes. 


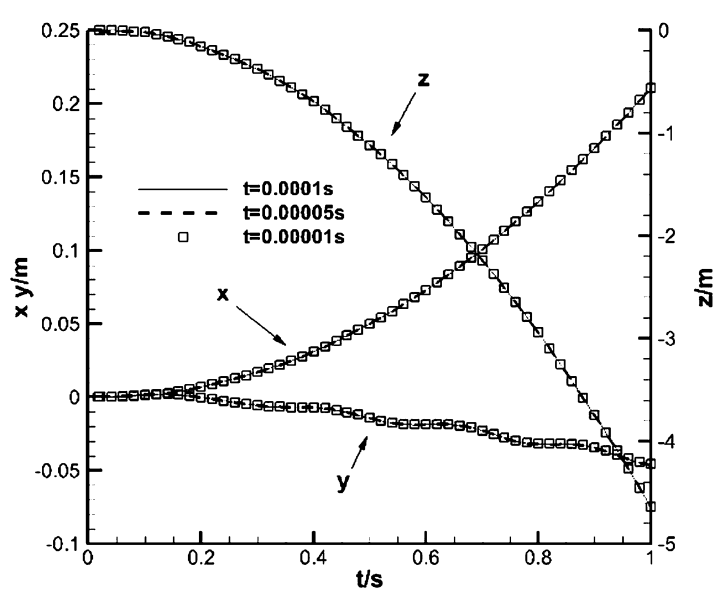

(a) The displacement responses of the store

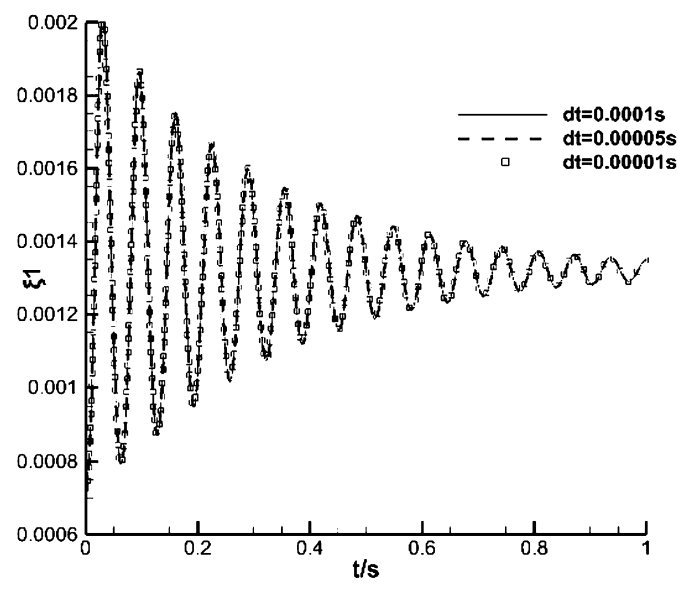

(c) Generalized displacement response $\xi 1$ of the carrier

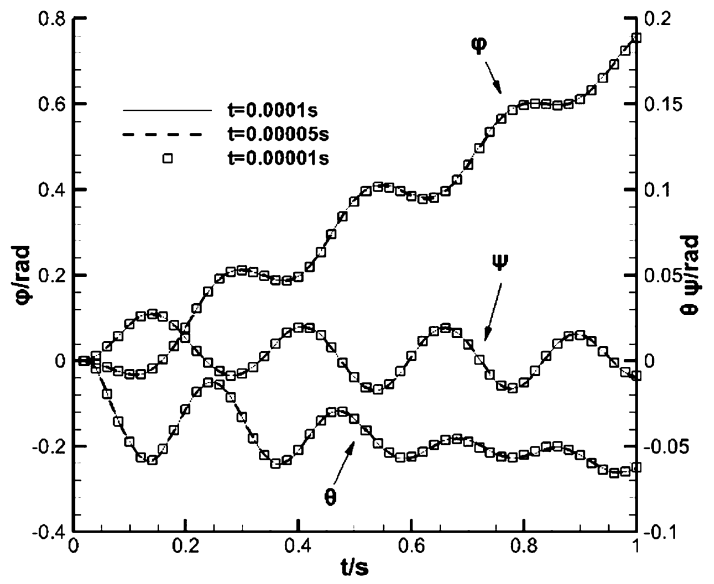

(b) The attitude angle responses of the store

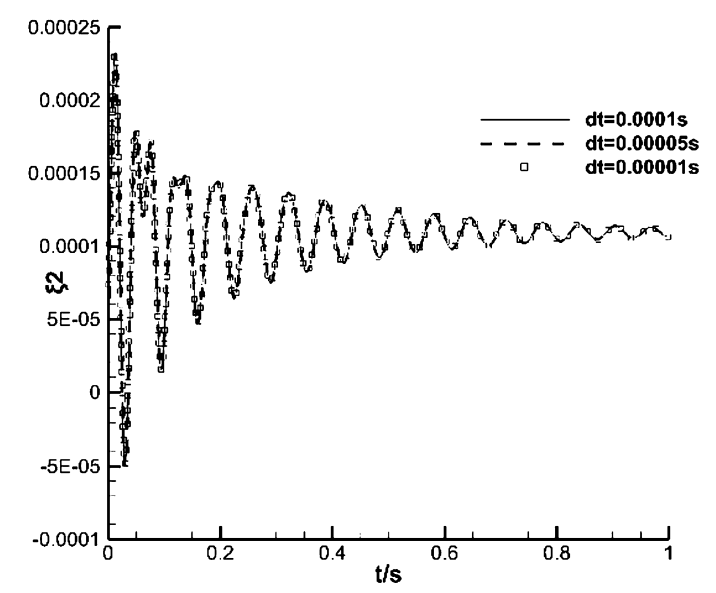

(d) Generalized displacement response $\xi 2$ of the carrier

Fig. 11. The dynamic responses of the store and the carrier for different time step sizes.

Table 3

The accumulated error estimates.

\begin{tabular}{llll}
\hline $\begin{array}{l}\text { Simulation } \\
\text { method }\end{array}$ & $\begin{array}{l}\text { Time step number } \\
\text { of CFD }\end{array}$ & $\begin{array}{l}\text { Allowable number of time steps (allowable } \\
\text { value of total error is 0.001) }\end{array}$ & $\begin{array}{l}\text { Accumulated } \\
\text { error }\end{array}$ \\
\hline MTS & 118 & 1166400 & 0.00001006 \\
CFD/CSD/RBD & 1000 & 1166400 & 0.00002928 \\
\hline
\end{tabular}

ulation method, the accumulated error is less than $35 \%$ of that of the CFD/CSD/RBD method.

\subsubsection{Comparison of computational efficiency}

In this section, the computational efficiency of the MTS ROM/ $\mathrm{CFD} / \mathrm{CSD} / \mathrm{RBD}$ method is studied in detail. For the above CFD/ CSD/RBD simulation, the physical time step size is $0.001 \mathrm{~s}$. It requires 1000 times of CFD calculations and about $2000 \mathrm{~h} \mathrm{CPU} \mathrm{time}$ to compute the dynamic responses of $1.0 \mathrm{~s}$ for one case. For the MTS ROM/CFD/CSD/RBD method, however, the CPU time of ROM training is only about $64 \mathrm{~h}$. Once the ROM is prepared, it can be used for a large number of cases. In this simulation, the time step size is selected as $0.001 \mathrm{~s}$ at the initial solution stage with a total time of $0.02 \mathrm{~s}$, and then the multi-time scale approach is employed. The time step sizes of CFD/RBD and RBD/CSD coupling calculations are $0.01 \mathrm{~s}$ and $0.0001 \mathrm{~s}$ respectively, and it only requires 118 times of CFD calculations and about $220 \mathrm{~h} \mathrm{CPU} \mathrm{time}$ for the same problem. The calculation amount is reduced by about an order of magnitude (see Table 4).
Table 4

The CPU time of the simulation with different methods.

\begin{tabular}{lll}
\hline Calculation method & & CPU time/h \\
\hline MTS ROM/CFD/CSD/RBD & ROM training & 64 \\
& Dynamic simulation & 220 \\
CFD/CSD/RBD & Dynamic simulation & 2000 \\
\hline
\end{tabular}

\section{Conclusions}

In the flight dynamic simulation of elastic aircraft, the structural modal frequency is much higher than that of the rigid-body mode. In order to better describe the high frequency characteristics, the physical time step size is set much smaller than the period of flight mechanics. Consequently, the efficiency of rigid body dynamic simulation is low. Focusing on this problem, the MTS ROM/CFD/CSD/RBD coupled simulation method is introduced in this paper. According to the research, conclusions can be drawn as follows: 


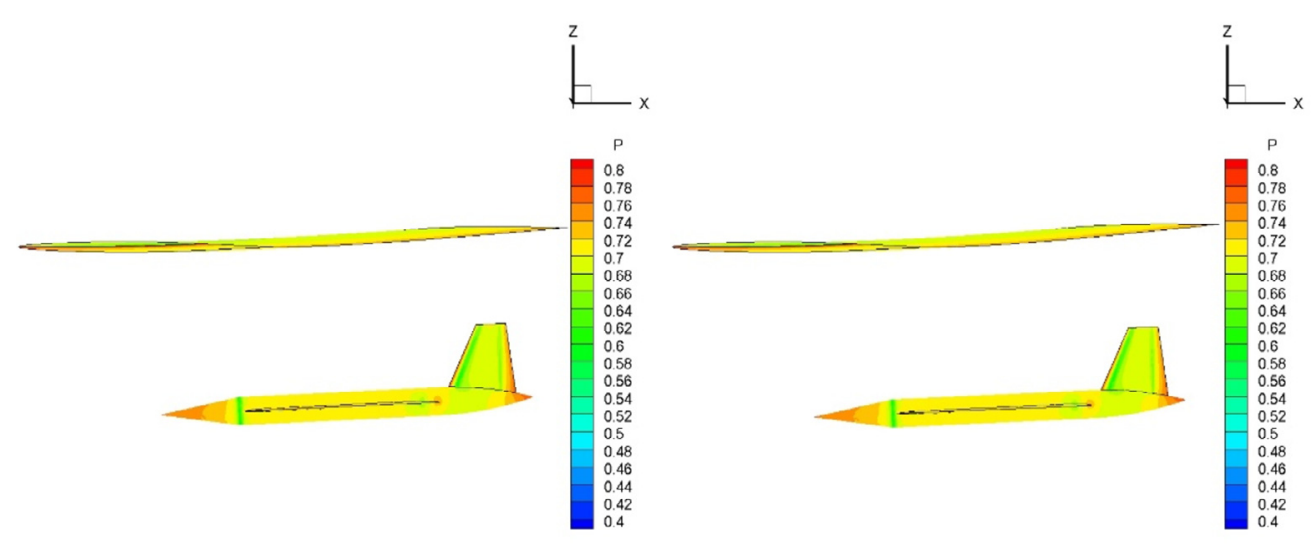

(a) $\mathrm{CFD} / \mathrm{CSD} / \mathrm{RBD}$ result at $\mathrm{t}=0.18 \mathrm{~s}$

(b) MTS ROM/CFD $/ \mathrm{CSD} / \mathrm{RBD}$ result at $\mathrm{t}=0.18 \mathrm{~s}$

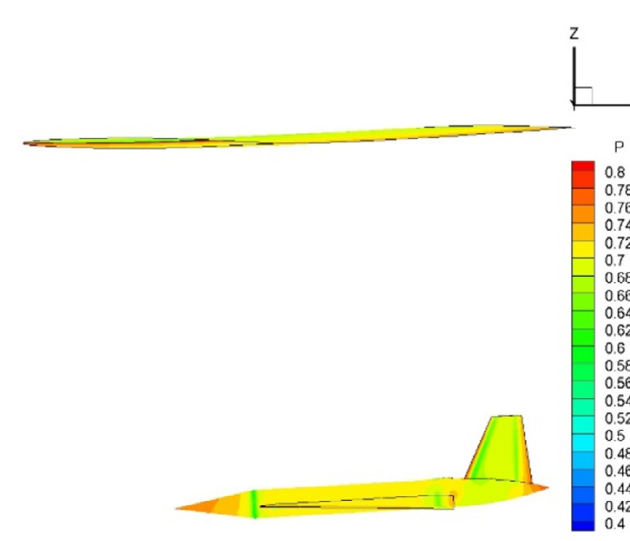

(c) $\mathrm{CFD} / \mathrm{CSD} / \mathrm{RBD}$ result at $\mathrm{t}=0.36 \mathrm{~s}$

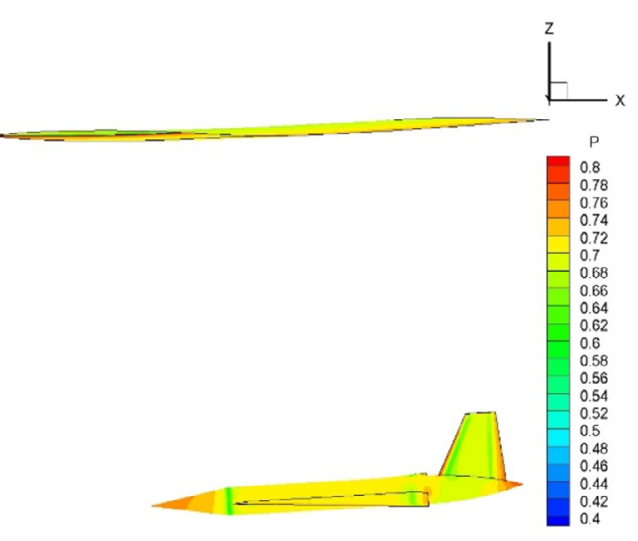

(d) $\mathrm{MTS} \mathrm{ROM} / \mathrm{CFD} / \mathrm{CSD} / \mathrm{RBD}$ result at $\mathrm{t}=0.36 \mathrm{~s}$

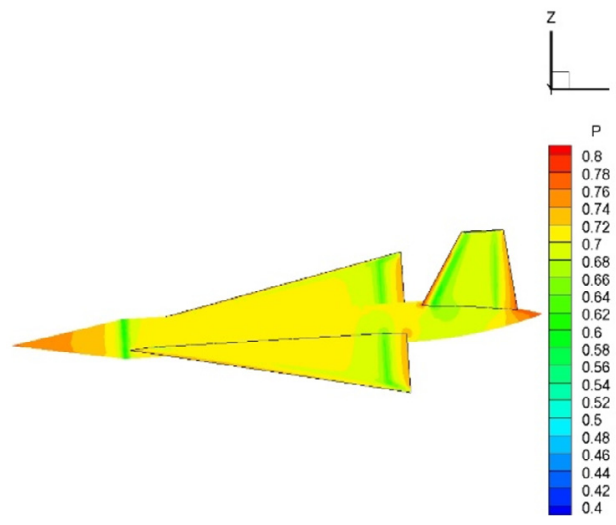

(e) $\mathrm{CFD} / \mathrm{CSD} / \mathrm{RBD}$ result at $\mathrm{t}=0.94 \mathrm{~s}$

(f) $\mathrm{MTS} \mathrm{ROM} / \mathrm{CFD} / \mathrm{CSD} / \mathrm{RBD}$ result at $\mathrm{t}=0.94 \mathrm{~s}$

Fig. 12. The comparison of the pressure contours results between the CFD/CSD/RBD and MTS ROM/CFD/CSD/RBD method at different times.

(1) In the dynamic simulation of store separation considering elastic carrier aircraft interference, the time responses of store obtained by the MTS ROM/CFD/CSD/RBD coupled simulation method have good agreement with those of the CFD/CSD/RBD results. The displacement and attitude angle relative errors are less than 3\% and 6\% respectively. Therefore, the proposed method has high precision.

(2) In the MTS ROM/CFD/CSD/RBD coupled simulation, the CPU time of ROM/CSD coupled simulation can be ignored. The calculation amount of this method is reduced by about an order of magnitude compared with the CFD/CSD/RBD coupled simulation method. The MTS ROM/CFD/CSD/RBD coupled simulation method can provide a solution strategy for similar engineering problems.

\section{Declaration of competing interest}

The authors declare that they have no known competing financial interests or personal relationships that could have appeared to influence the work reported in this paper. 


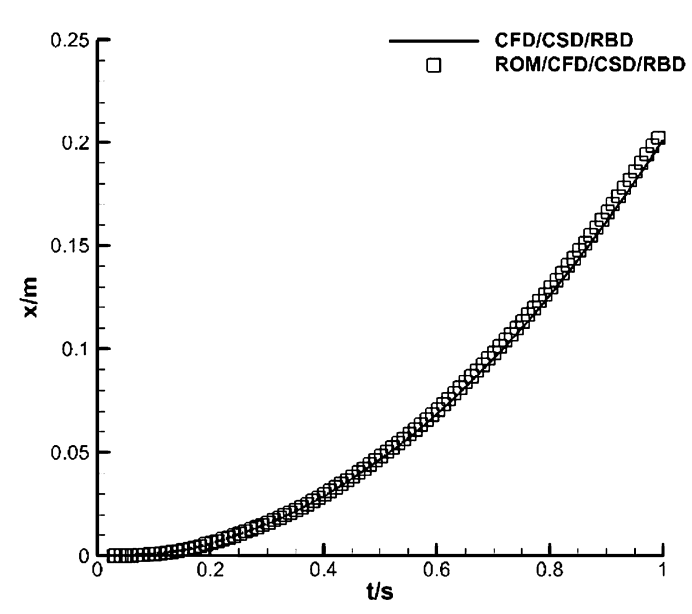

(a) Displacement response of the store in $\mathrm{x}$ direction

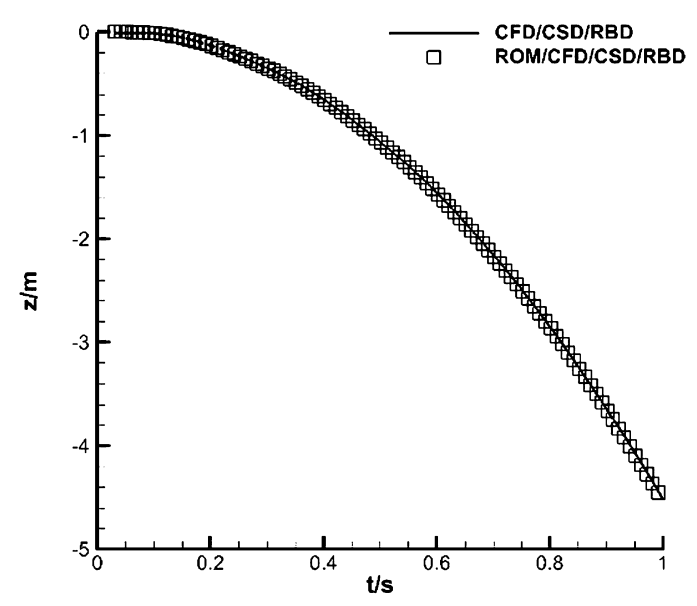

(c) Displacement response of the store in $\mathrm{z}$ direction

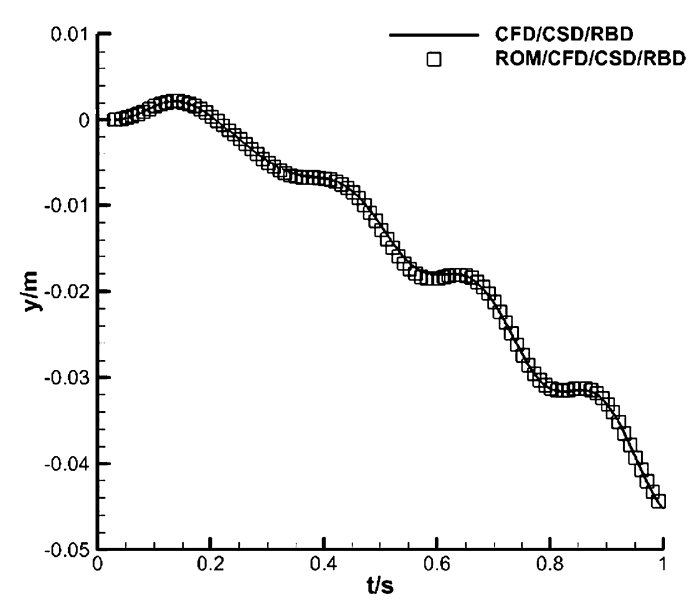

(b) Displacement response of the store in y direction

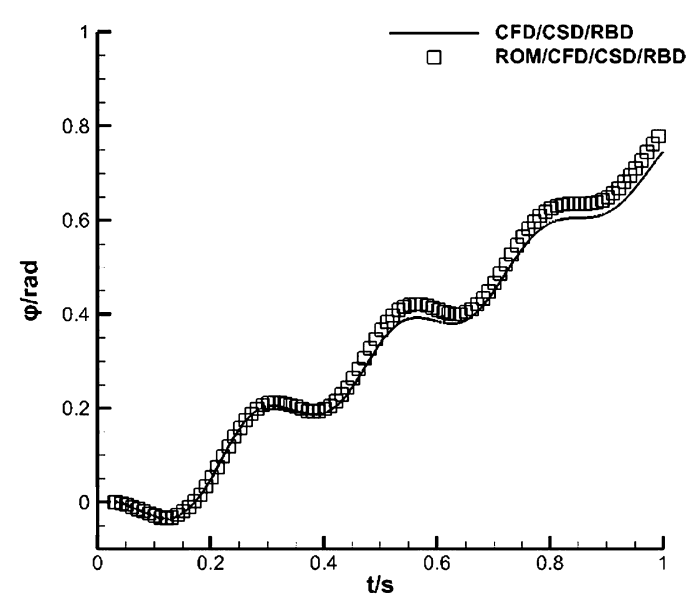

(d) Roll attitude response of the store $\varphi$

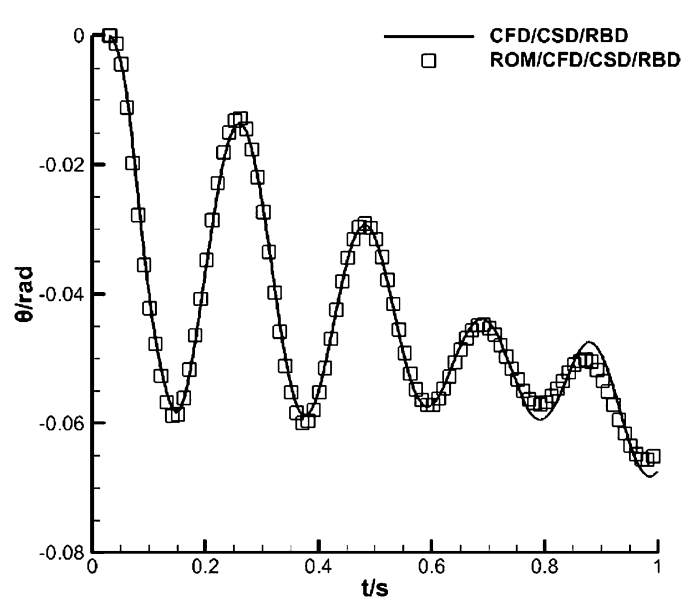

(e) Pitch attitude response of the store $\theta$

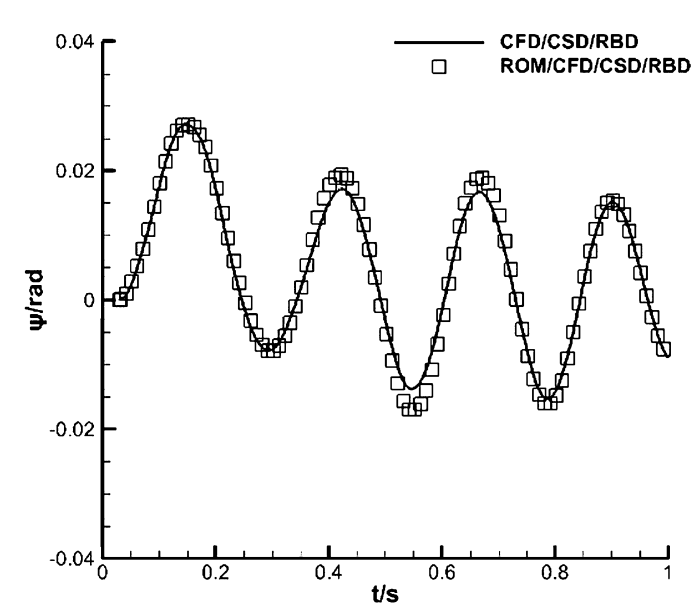

(f) Yaw attitude response of the store $\psi$

Fig. 13. The comparison of the dynamic responses results of the store between MTS ROM/CFD/CSD/RBD and CFD/CSD/RBD methods. 


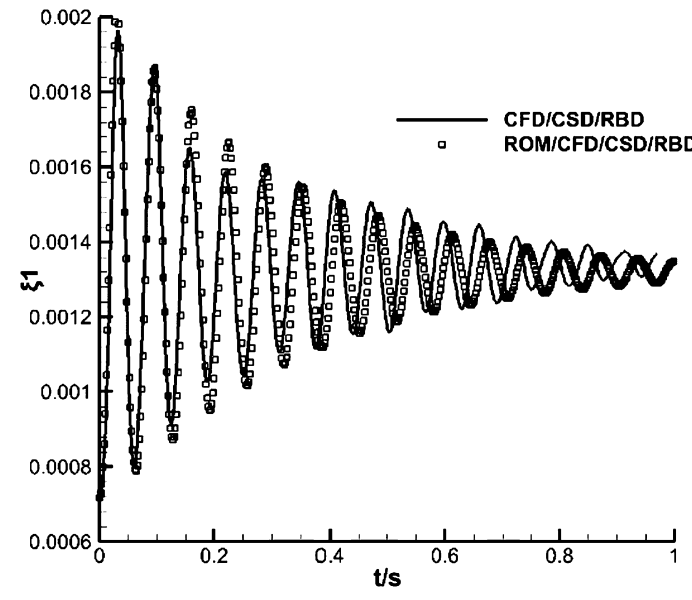

(a) Generalized displacement responses $\xi 1$ of the carrier

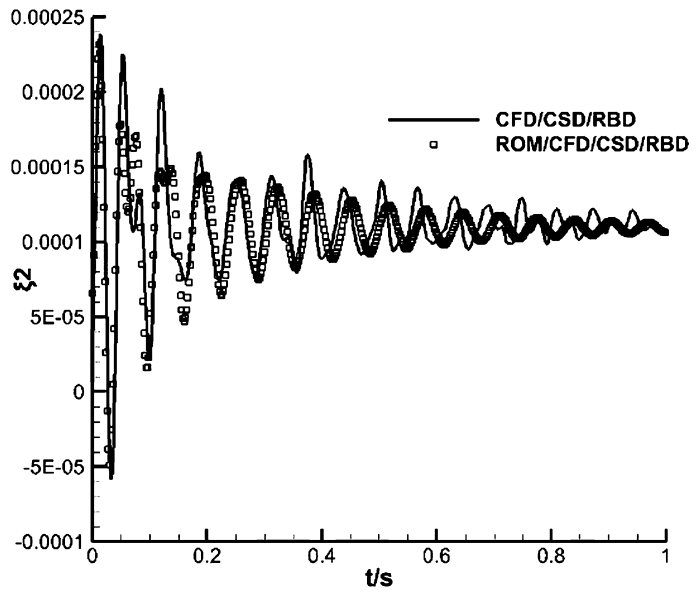

(b) Generalized displacement responses $\xi 2$ of the carrier

Fig. 14. The comparison of the generalized displacement responses results of the carrier between MTS ROM/CFD/CSD/RBD and CFD/CSD/RBD methods.

\section{Acknowledgements}

This work is supported by the Strategic Priority Research Program of Chinese Academy of Sciences (Grant No. XDA17030100).

\section{References}

[1] S. Henri, P.L. Helene, J.L. Farges, Analysis and optimization of an air-launch-toorbit separation, Acta Astronaut. 108 (2015) 18-29.

[2] N.S. Klijn, M.S. Klijn, C. Noel, Air-launching Earth to orbit: effects of launch conditions and vehicle aerodynamics, J. Spacecr. Rockets 42 (2005) 569-572.

[3] R.H. Hua, C.X. Zhao, Z.Y. Ye, et al., Effect of elastic deformation on the trajectory of aerial separation, Aerosp. Sci. Technol. 45 (2015) 128-139.

[4] L. Yang, Z.Y. Ye, The interference aerodynamics caused by the wing elasticity during store separation, Acta Astronaut. 121 (2016) 116-129.

[5] M.T. Kozak, e.N. Yildiz, Y. Yazicioglu, et al., Effects of aircraft aeroelastic deformations on external store separation dynamics, Paper IMECE 2013-63772.

[6] K. Anandhanarayanan, K. Arora, V. Shah, et al., Separation dynamics of air-toair missile using a grid-free Euler solver, J. Aircr. 50 (2013) 725-731.

[7] S.K. Marti, S.K. Nesrin, M. Bob, et al., Flight testing of a new Earth-to-orbit airlaunch method, J. Aircr. 43 (2006) 577-583.

[8] D.C. Zhang, Towed Reusable Launch Vehicle Flight Dynamics, Northwestern Polytechnical University, 2005.

[9] J.M. Lee, K.S. Dunworth, D.J. Atkins, Studies of combined use of CFD and wind tunnel test approaches to simulate a store separation from F-15E using efficient CFD database generation, Paper AIAA 2004-4724.

[10] R.C. Snyder, W.E. Roberts, Application of platform knowledge and adaptation of store separation analysis and testing to multiple-carriage weapon suspension systems: a case study, in F-16/BRU-57, Paper AIAA 2006-454.

[11] M. Rizk, B. Jolly, Aerodynamic simulation of bodies with moving components using CFD overset grid methods, Paper AIAA 2006-1252.

[12] P.A. Cavallo, S.M. Dash, Aerodynamics of multi-body separation using adaptive unstructured grids, Paper AIAA 2000-4407.

[13] G. Wang, X. Chen, Y. Xing, Z. Zeng, Multi-body separation simulation with an improved general mesh deformation method, Aerosp. Sci. Technol. 71 (2017) $763-771$.

[14] L. Yang, Z.Y. Ye, J. Wu, The influence of the elastic vibration of the carrier to the aerodynamics of the external store in air-launch-to-orbit process, Acta Astronaut. 128 (2016) 440-454.

[15] R.H. Hua, Z.Y. Ye, J. Wu, Effect of elastic deformation on flight dynamics of projectiles with large slenderness ratio, Aerosp. Sci. Technol. 71 (2017) 347-359.

[16] A.T. Nguyen, J.H. Han, Wing flexibility effects on the flight performance of an insect-like flapping-wing micro-air vehicle, Aerosp. Sci. Technol. 79 (2018) 468-481.
[17] W.W. Zhang, Z. Lv, Q.Q. Diwu, H.S. Zhong, A flutter prediction method with low cost and low risk from test data, Aerosp. Sci. Technol. 86 (2019) 542-557.

[18] M. Winter, F.M. Heckmeier, C. Breitsamter, CFD-based aeroelastic reduced-order modeling robust to structural parameter variations, Aerosp. Sci. Technol. 67 (2017) 13-30.

[19] W.W. Zhang, K.J. Chen, Z.Y. Ye, Unsteady aerodynamic reduced-order modeling of an aeroelastic wing using arbitrary mode shapes, J. Fluids Struct. 58 (2015) 254-270.

[20] W.W. Zhang, Z.Y. Ye, C. Zhang, ROM based aeroservoelastic analysis in transonic flow, J. Aircr. 46 (2009) 2178-2183.

[21] X.T. Li, Y.L. Liu, J.Q. Kou, et al., Reduced-order thrust modeling for an efficiently flapping airfoil using system identification method, J. Fluids Struct. 69 (2017) 137-153.

[22] J.Q. Kou, W.W. Zhang, A hybrid reduced-order framework for complex aeroelastic simulations, Aerosp. Sci. Technol. 84 (2019) 880-894.

[23] R. Huang, H.J. Liu, Z.J. Yang, et al., Nonlinear reduced-order models for transonic aeroelastic and aeroservoelastic problems, AIAA J. 56 (2018) 3718-3731.

[24] R. Huang, H.K. Li, H.Y. Hu, et al., Open/closed-loop aeroservoelastic predictions via nonlinear, reduced-order aerodynamic models, AIAA J. 53 (2015) $1812-1824$.

[25] J. Blazek, Computational Fluid Dynamics: Principles and Applications, Elsevier, UK, 2005

[26] R.H. Hua, X.X. Yuan, Z.G. Tang, et al., Study on flight dynamics of flexible projectiles based on closed-loop feedback control, Aerosp. Sci. Technol. 90 (2019) 327-341.

[27] Y.W. Jiang, Algebraic-volume meshfree method for application in finite volume solver, Comput. Methods Appl. Mech. Eng. 355 (2019) 44-66.

[28] Y.W. Jiang, Numerical Solution of Navier-Stokes Equations on Generalized Mesh and Its Applications, Northwestern Polytechnical University, 2013.

[29] W.W. Zhang, Z.Y. Ye, Control law design for transonic aeroservoelasticity, Aerosp. Sci. Technol. 11 (2007) 136-145.

[30] T.J. Cowan, Efficient Aeroelastic CFD Predictions Using System Identification, Oklahoma State University, 1996.

[31] E.C. Yates, AGARD standard aeroelastic configurations for dynamic response Iwing 445.6, AGARD Report No. 765, 1988.

[32] N.N. Smirnov, V.B. Betelin, V.F. Nikitin, et al., Accumulation of errors in numerical simulations of chemically reacting gas dynamics, Acta Astronaut. 117 (2015) 338-355.

[33] N.N. Smirnov, V.B. Betelin, R.M. Shagaliev, et al., Hydrogen fuel rocket engines simulation using LOGOS code, Int. J. Hydrog. Energy 39 (2014) 10748-10756. 